\title{
Transition to Instability in Segmented Rijke Tube
}

\author{
Rafael Hernandez and Konstantin I. Matveev ${ }^{1, *}$
}

\author{
Washington State University, Pullman, WA, USA
}

\begin{abstract}
Thermoacoustic instabilities can arise in systems where unsteady heat release is favorably coupled with acoustic pressure oscillations. A modified Rijke tube with segments having different sections is found to have lower threshold levels of heat addition rate needed for exciting the fundamental acoustic mode of the tube. Experiments indicate that about $115 \mathrm{~W}$ of heating power is required to produce sound in the $90-\mathrm{cm}$-long segmented tube, while over $230 \mathrm{~W}$ is needed in 60 -cm-long and 90-cm-long tubes with constant-area cross sections. One of the causes for lowering the threshold of heat addition rate is due to a significant reduction of the resonator natural frequency. The presented results suggest an importance of including the system geometry details into analysis of practical devices prone to thermoacoustic instabilities.
\end{abstract}

Keywords: Rijke tube, thermoacoustics, resonator, acoustic instability.

\section{INTRODUCTION}

Self-excited acoustic oscillations can appear in resonators with unsteady heat release. Many newly developed rocket motors and industrial burners experience these instabilities [1]. Acoustic oscillations can lead to the system performance degradation, loud noise, and even damage of these devices. One of the main instability mechanisms is due to a coupling of sound waves and unsteady heat release. If extra heat is supplied to the gas during its compression phase in an acoustic cycle, then a time-averaged thermal-to-acoustic energy conversion occurs according to Rayleigh criterion [2].

Thermoacoustic instabilities can be studied experimentally in a simple system known as a Rijke tube [3]. It represents an open-ended tube with a mesh inside (Fig. 1a), which is heated by flame or electrically. In the original version the tube was oriented vertically, so that a mean gas flow in the tube was provided by natural convection. The main excitation mechanism is due to a time delay between an instantaneous velocity at the heater and the time-dependent heat release. If small flow fluctuations are present the tube and the time delay ensures positive thermoacoustic energy conversion (i.e., Rayleigh criterion is satisfied), then fluctuations can grow into high-amplitude acoustic oscillations. The fundamental acoustic mode of the tube is excited when the mesh is heated to a sufficiently high temperature and positioned at about the quarter-tube-length distance from the upstream tube end. (Higher modes can be excited at other mesh locations, but require higher mesh temperatures). Later modifications of the Rijke tube included a horizontal orientation with a mean flow provided by other means, such as a blower [4]. In such a setup, an operational range of the system parameters is significantly extended, since mean flow is controlled independently of heat addition rate.

*Address correspondence to this author at the MME School, WSU, Pullman, WA, 99164-2920, USA; Tel: +1-509-3051327; Fax: +1-509-3054662; E-mail: matveev@wsu.edu
In previous studies of Rijke tubes, constant-section tubes were commonly used [5]. However, practical systems prone to thermoacoustic instabilities have more complex geometries. In the present study, two circular tubes with different cross-sectional areas are connected together to form a single resonator (Fig. 1b). It appears that the sound onset in this system can occur at significantly lower levels of supplied heating power. The experimental setup and results are presented below.

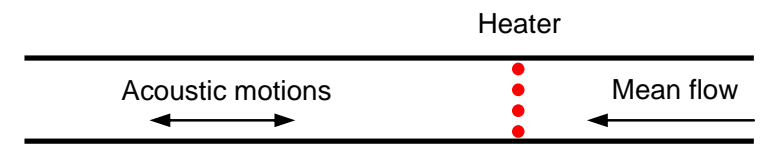

a

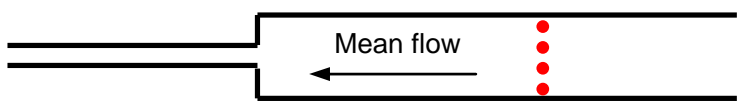

b

Fig. (1). Schematics of Rijke tubes: (a) standard and (b) segmented.

\section{EXPERIMENTAL SYSTEMS}

The structure of the experimental apparatus is shown in Fig. (2). The segmented Rijke tube in this system consists of an upstream aluminum tube with internal diameter $5.2 \mathrm{~cm}$ and length $60 \mathrm{~cm}$ and a downstream steel tube with internal diameter $2.4 \mathrm{~cm}$ and length $30 \mathrm{~cm}$. In additional tests, a constant-area tube was applied to provide reference data of a classical single-segment Rijke tube setup.

The mean air flow through the tube is provided by an electrically regulated Jabsco 35440 blower. To exclude the influence of natural convection on the flow rate, the horizontal orientation of the Rijke tube was implemented. A buffer chamber, located between the tube and the blower, is intended to suppress interactions between the blower and tube 


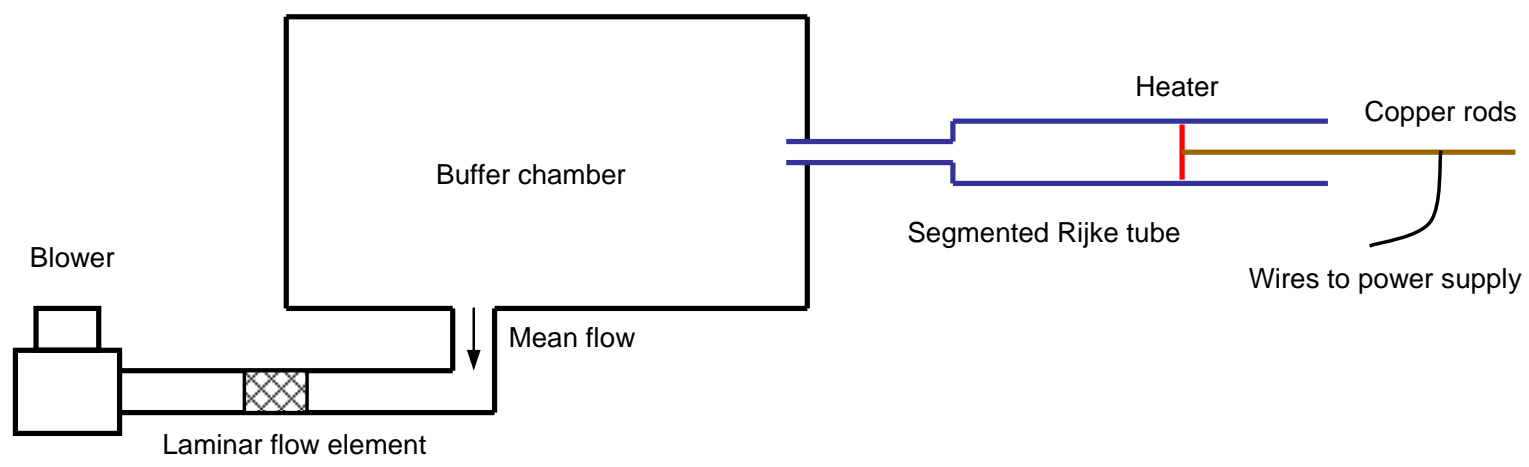

Fig. (2). Experimental setup (shown not to scale).

acoustics. The chamber dimensions are $46 \times 46 \times 76 \mathrm{~cm}$; and its internal surface is covered by 2.5 -cm-thick soundabsorbing polyurethane foam. The mean airflow rate is measured by a custom-built laminar flow element installed between the buffer chamber and the blower. The pressure drop is measured by a Dwyer Magnehelic Pressure Gage 2000. The laminar flow element was calibrated against a Dwyer variable-area flowmeter RMA-10. The temperature of the flow through the laminar flow element was monitored in the tests in order to determine the actual flow rates.

The heat source is an electrically powered nichrome wire of diameter $1.0 \mathrm{~mm}$ and length $75 \mathrm{~cm}$. The wire is mounted on a frame made of alumina silicate ceramic to minimize thermal and avoid electrical contacts with the tube walls. A photo of the heating element is shown in Fig. (3). Copper rods of diameter $0.32 \mathrm{~cm}$ are applied for feeding the nichrome wire with electric current from a Sorensen DCS 33-36E power supply.

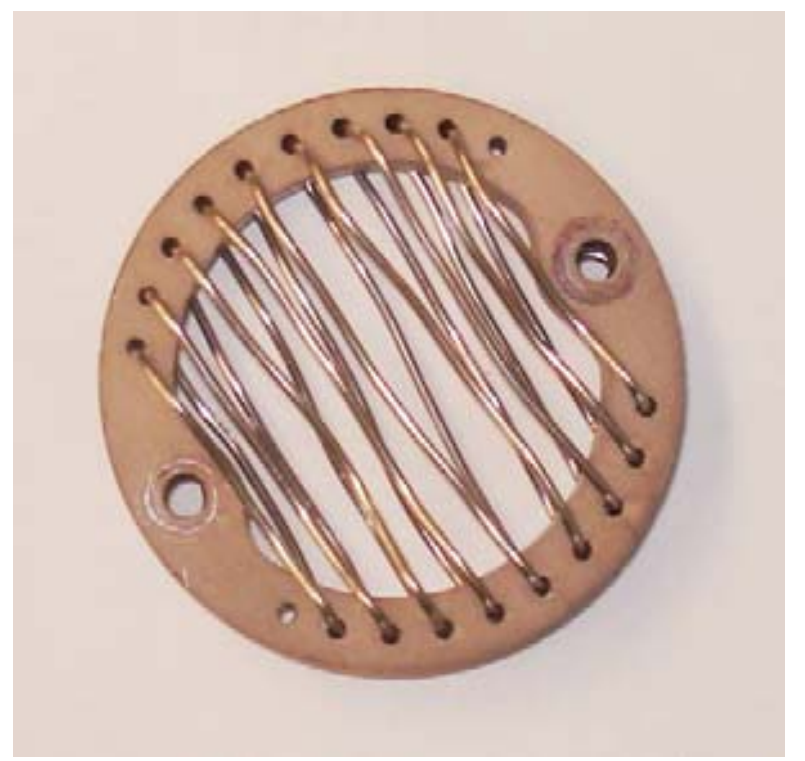

Fig. (3). Heating element: nichrome wire mounted on ceramic ring.

\section{RESULTS}

The first test sequence was conducted with a singlesegment, 5.2-cm-diameter tube (as shown in Fig. 1a) with a purpose to obtain reference data in a standard Rijke tube setup. In the preliminary tests, the heater position most favorable for generating sound was determined: $12.5 \mathrm{~cm}$ from the upstream tube end. Further tests in this tube were conducted at this position of the heater. Experimental runs were carried out at different mean flow rate settings. In each test, an electric power supplied to the heater was slowly increased with a rate of change of about $2 \mathrm{~W} / \mathrm{min}$. At a certain level of power, an audible tonal sound appeared, corresponding to the transition to thermoacoustic instability. The stability boundary obtained in a single-segment tube is shown by circles in Fig. (4). There is an optimal mean flow rate at which the power required to produce sound has the lowest value. With increasing or decreasing the mean flow rate, the threshold power increases. This behavior is similar to previously observed stability boundaries in Rijke tubes $[4,5]$. The frequency of acoustic oscillations measured by an external microphone at the mean flow rate $0.95 \mathrm{~g} / \mathrm{s}$ is about $310 \mathrm{~Hz}$, which corresponds to the natural frequency of the tube fundamental mode.

Practical devices where thermoacoustic instabilities are a concern have more complex geometries than a singlesegment tube. Altered system geometries may lead to earlier transitions to instability requiring lower power released at the heater. One of such modifications is a double-segment tube with the downstream section having a reduced crosssectional area (Fig. 1b). One of the factors that can encourage early transition to instability is a decreased natural frequency of the system, since acoustic losses in the resonator increase with frequency. A segmented tube has a natural frequency lower than that in a tube of the same length, while the surface area in a segmented tube does not increase as much as in a simply lengthened tube with the same diameter. Additionally, a recirculation zone in the mean flow in front of the transition between tube segments may also serve a source of instabilities and encourage earlier sound onset. Variable-area resonators are also known to ensure earlier transition to instability in other types of thermoacoustic devices that do not involve mean flow, such as standing-wave thermoacoustic engines [6].

The most favorable position of the heater for sound generation in a double-segment Rijke tube was experimentally found to be at $35 \mathrm{~cm}$ from the tube upstream end. The threshold power values recorded at the sound onset in this configuration are given by squares in Fig. (4). The minimum power required for the transition to instability drops nearly twice in comparison with a single-segment tube (Fig. 4), 


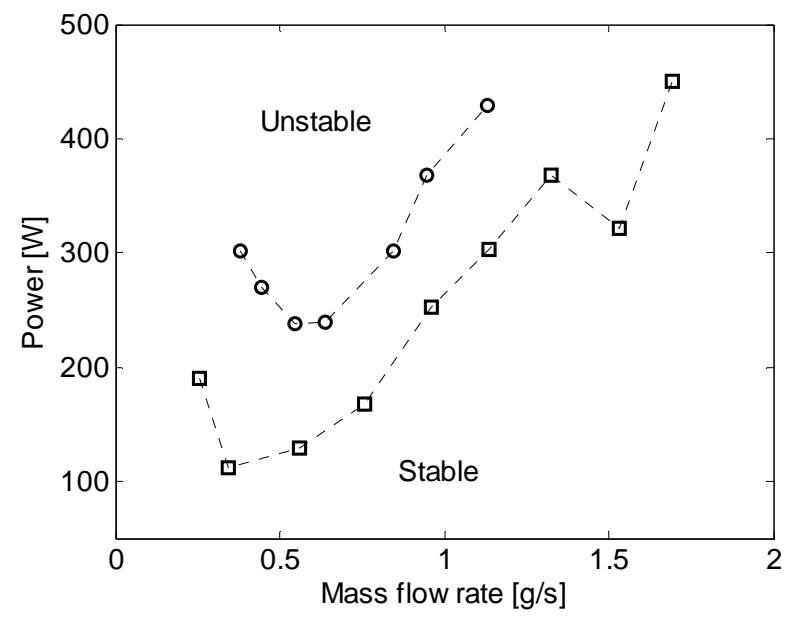

Fig. (4). Minimum electric power needed for generating sound in studied Rijke setups: circles, constant-area tube; squares, segmented tube.

while the range of mean flow rates where instability is possible at available heating power expands significantly. A secondary local minimum in the threshold power data is observed at the flow rate of $1.53 \mathrm{~g} / \mathrm{s}$. The sound frequency measured after the sound onset at the mean flow rate $0.95 \mathrm{~g} / \mathrm{s}$ is about $165 \mathrm{~Hz}$.

A tube with the constant diameter $5.2 \mathrm{~cm}$ and length 90 $\mathrm{cm}$ (same as the total length of the double-segment tube) was also tried. However, it was discovered that the lowest power necessary for the sound appearance (about $300 \mathrm{~W}$ ) exceeded that of the short tube, while the frequency of the excited sound was about $200 \mathrm{~Hz}$. This confirms a strong effect of the tube segmentation on the required heating power to achieve thermoacoustic instability.

\section{CONCLUDING REMARKS}

Experiments conducted with a segmented Rijke tube demonstrate that about twice lower levels of supplied heating power are needed for generating sound in comparison with a classical single-segment Rijke tube. The range of flow rates suitable for sound generation is also significantly greater. These effects may be caused by reduced acoustic losses in the modified system. The obtained results suggest that a sufficient level of details about system geometries must be included into consideration when analyzing practical devices prone to this sort of instabilities. Another implication of this study is for simplifying Rijke-tube lecture demonstrations: a segmented tube would need much less heating power to produce sound.

\section{ACKNOWLEDGEMENTS}

This material is based upon work supported by the National Science Foundation under Grant No. 0853171.

\section{REFERENCES}

[1] F. E. C. Culick, Unsteady Motions in Combustion Chambers for Propulsion Systems, Neuilly-sur-Seine Cedex, France: NATO/RTO AGARDograph AG-AVT-039, NATO/RTO, 2006.

[2] J. W. S. Rayleigh, The Theory of Sound, Dover Publications, 1945.

[3] P. L. Rijke, "Notiz über eine neie art, die luft in einer an beiden enden offenen Röhre in schwingungen zu versetzen", Annalen der Physik, vol .107, pp. 339-343, 1859

[4] K. I. Matveev and F. E. C. Culick, "A Study of the Transition to Instability in a Rijke Tube with Axial Temperature Gradient," $J$. Sound Vib., vol. 264, pp. 689-706, 2003.

[5] R. L. Raun, M. W. Beckstead, J. C. Finlinson, and K. P. Brooks, "A review of Rijke tubes, Rijke burners and related devices", Prog. Energ. Combust., vol. 19, pp. 313-364, 1993.

[6] G. W. Swift, Thermoacoustics: A Unifying Perspective for Some Engines and Refrigerators, Sewickley, PA: Acoustical Society of America, 2002. 